\title{
A Comparative Study of Take-Off Technique for the Excellent Male Long Jump in Shandong and the World
}

\author{
Zhenxiang Tian, Degang $\mathrm{Xu}^{*}$ \\ Zaozhuang University
}

\begin{abstract}
This article takes of eight men's long jump athletes of the track team Shandong province as the research object, using the image analysis, mathematical statistics, comparative analysis and other methods, comparative analysis the parameters of the long jump take-off technology with excellent athletes in the world. The results show that the take-off angle of Shandong athletes is smaller than the world's, and the jump horizontal speed is generally low, and the vertical speed of take-off is still to be improved. Suggested that we should pay attention to develop the absolute speed, increase the ratio of vertical speed in the initial velocity of take off, suggested increasing the core strength training on the basis of regular strength training, in order to improve the waist and abdomen and lower limb muscle strength, improve the take-off technology action and improve performance.
\end{abstract}

Keywords-Male Long jump; Take-off; Velocity; Angle

\section{INTRODUCTION}

Long jump is one of the oldest events in track and field. The modern long jump began in the 18th century in Britain, and flourished with the rise and development of the modern Olympic movement. In the course of many years, the long jump has made profound changes in technology and training methods. From all over the world of sports scientific research personnel of the project, each technical links from different angles and aspects of analysis and research, reveals the results of many with universality and regularity, for the continuous development of the project, has played a very good guide and push forward. In the last century, the theory and practice of the theory and practice have proved the rationality of this technique.

The complete long jump technology is composed of run-up, take-off, vacated and landing four consecutive action. Long jump belongs to the scope of oblique throwing [1]. Take-off technique is the most complicated technique in the whole longjump exercise. Therefore, this paper makes a biomechanical analysis on the take-off process of the elite male long-jump athletes in Shandong Province so as to accurately analyze and evaluate their movement skills and find a breakthrough to solve the teaching and training problems, to explore the inherent relationships and rules among various technical parameters during take-off, so as to provide the reference for realizing the optimal combination of take-off techniques for Chinese male long-jump athletes.

\section{TAKE-OFF STAGE ACTION ANALYSIS}

\section{A. The Angle of take-off technology}

The take-off technology includes the following angles, which include the landing angle, knee angle, leg drive angle and rising angle. Landing angle refers to the instant moment of the foot on the floor, the angle between the line connecting the ankle and body center of gravity and the horizontal plane. Landing angle for the world's excellent athletes is generally $66^{\circ} \pm 3$ [2]. The knee angle has been changing throughout the course of take-off, and most people take the perspective of three phases in the study: the moment of landing, the moment of maximum cushioning and the moment of leaving the ground Studies have shown that the world's excellent athletes respectively $166^{\circ}, 144^{\circ}, 173^{\circ}$ [2]. Leg drive angle refers to the moment when the long jumper takes off from the ground, the angle between the line connecting the ankle and body center of gravity and the horizontal plane. Many think most appropriate leg drive angle is about $71^{\circ}-77^{\circ}$ [3]. The rising angle is the angle between the center of gravity and the horizontal plane at the moment of departure from the foot during take-off. It can not be directly measured as the other angles but must be calculated through the speed. It depends on the horizontal speed obtained during the run-up and the vertical speed obtained during the stepping. With the increase of the rising angle, it will reduce the speed of rising and closing, and increase the rising angle within a reasonable range to get bigger rising height. With the increase of the rising angle, it will reduce the speed of rising and closing, and increase the rising angle within a reasonable range to get bigger rising height. According to previous statistics, the rising angles of outstanding foreign athletes average $21.9^{\circ}$ [4].

\section{B. Take-off time}

Take-Off time refers to the time between the moment when the jump-off foot moves to the ground. Under the premise of complete take-off action, the take-off time is negatively correlated with the runway speed and the long jump performance. The correlation coefficients are -0.53 and -0.69 respectively [5]. 


\section{Take-off stage speed}

The speed of the stage of take-off refers to the speed of the center of gravity of the athlete and is divided into two directions, horizontal and vertical. Studies have shown that the horizontal velocity of moment of gravity of long-jump sports has a highly positive correlation with the effective score, the correlation coefficient is 0.846 [5]. The vertical speed of the center of gravity during take-off is mainly derived from the horizontal speed and the pull-out strength of the take-off and landing. The decrease of the horizontal speed leads to another index, the horizontal speed loss, which refers to the decrease of the horizontal speed caused by the braking of the body during take-off [6]. Data show that the average rate of loss of athletes in China is $9.24 \%$, the world's best athletes is $10.27 \%$ [7].

TABLE I. CHINESE AND FOREIGN OUTSTANDING MALE LONG JUMPER TAKE-OFF STAGE BODY CENTER OF GRAVITY SPEED COMPARISON LIST (M / S) [8]

\begin{tabular}{|c|c|c|c|c|c|}
\hline & \multirow{2}{*}{$\begin{array}{c}\text { On the ground } \\
\text { horizontal } \\
\text { velocity }\end{array}$} & \multicolumn{2}{|c|}{ Take off from the ground } & \multirow{2}{*}{$\begin{array}{l}\text { the initial velocity of } \\
\text { take off, }\end{array}$} & \multirow{2}{*}{$\begin{array}{c}\text { horizontal speed } \\
\text { loss }\end{array}$} \\
\hline & & $\begin{array}{l}\text { horizontal sub- } \\
\text { speed }\end{array}$ & $\begin{array}{l}\text { velocity sub- } \\
\text { speed }\end{array}$ & & \\
\hline Chinese & $9.78 \pm 0.33$ & $8.89 \pm 0.34$ & $3.10 \pm 0.25$ & $9.41 \pm 0.26$ & $0.89 \pm 0.22$ \\
\hline Foreigner & $10.66 \pm 0.28$ & $8.83 \pm 0.38$ & $3.41 \pm 0.25$ & $9.36 \pm 0.34$ & $1.83 \pm 0.75$ \\
\hline
\end{tabular}

\section{RESEARCH METHODS}

\section{A. Research object}

In this study, a total of seven excellent male long-jump athletes in Shandong long-jump sports team were tested, the basic situation in Table 2 .

TABLE II. BASIC SITUATION OF THE SUBJECTS

\begin{tabular}{cccc}
\hline Name & Age & Height/cm & Best result $/ \mathrm{m}$ \\
\hline 1 & 26 & 190 & 8.02 \\
2 & 19 & 191 & 7.28 \\
3 & 21 & 183 & 7.65 \\
4 & 27 & 190 & 7.90 \\
5 & 23 & 185 & 7.88 \\
6 & 24 & 186 & 7.86 \\
7 & 25 & 180 & 7.86 \\
8 & 19 & 182 & 7.45 \\
\hline
\end{tabular}

\section{B. Test procedures}

\section{1) Equipment and site Settings}

The test was conducted at the training hall with wind speed of zero. Two Sony digital cameras $(\mathrm{C} 1, \mathrm{C} 2)$ are placed on the right, $20 \mathrm{~m}$ from the runway, and each machine has a shooting range of about $6 \mathrm{~m}$ on the runway. The shooting frequency of the camera is $50 \mathrm{~Hz}$, the shutter speed is $1 / 250$ s and the tripod height is $1.5 \mathrm{~m}$.

\section{2) Test Procedure}

- The testers asked for the subject details and numbered them;

- Subjects warm-up exercise, testers placed equipment;

- Shooting scale, camera position will not be able to change after shooting scale;

- Athletes according to the number of test jump, each athlete try six times, and began shooting;

- Finishing shooting equipment finishing.

\section{Data collection}

\section{1) Film cutting}

Trim the program using the APAS software for digital movie editing. Trim software can be AVI format film frameby-frame, arbitrary editing of each frame.

\section{2) Digitize}

Digitized by APAS Digitize program, manually calibrated each of the body mark points (17 in total) by frame with cross cursor, and obtained the coordinates of 17 points by reading the scale file and the digital operation of the software, and then proceeded with the data Digital filtering, cut-off frequency of $10 \mathrm{~Hz}$.

\section{3) Data Extraction (Display)}

After the digitization step, APAS data is extracted to extract the required data. The angle indicator is obtained by Dartfish software.

4) Mathematical Statistics

The use of SPASS18.0 software for statistical data processing. 
5) Comparative analysis

Compare the collected data with the data of the world's elite athletes and draw a conclusion

\section{RESULT AND ANALYSIS}

\section{A. Analysis of the parameters of take-off technology}

The take-off action takes place through the cushioning and pedaling of the leg of the take-off leg so as to generate an effective vertical momentum for the momentum of the run-up so that the body regains a larger upward vertical velocity, its task is in the case of minimizing the loss of horizontal velocity as far as possible, to create a higher vertical speed, so that athletes get higher initial speed. As can be seen from the comparison between Table 1 and Table 3, the gaps with the world's top athletes on the horizontal speed and the groundbased horizontal speed are not great, but the vertical speeds of the jumping-off are quite different, indicating that they are fast The ability to take a quick take-off under the pitch needs to be improved. If the speed of the run-up is improved, the long jump result will be further improved. It is suggested that special attention should be paid to improve the vertical speed during take-off and to increase or maintain the horizontal speed of take-off and increase the proportion of vertical speed in initial speed, so as to improve takeoff movement technology, improve the ability of fast jump, more effectively improve the performance, to narrow the gap with the world elite athletes.

TABLE III. THE DATA OF SUBJECTS VELOCITY DURING TAKE-OFF PHASE OF EACH PHASE

\begin{tabular}{ccccc}
\hline \multirow{2}{*}{ Number } & $\begin{array}{c}\text { ground-based } \\
\text { speed }(\mathrm{m} / \mathrm{s})\end{array}$ & \multicolumn{2}{c}{ Jump off the ground speed $(\mathrm{m} / \mathrm{s})$} & \multirow{2}{*}{ loss of velocity } \\
\cline { 3 - 4 } & $10.93 \pm 0.14$ & $9.11 \pm 0.37$ & $2.89 \pm 0.46$ & $1.82 \pm 0.26$ \\
& $9.91 \pm 0.18$ & $8.67 \pm 0.09$ & $3.17 \pm 0.01$ & $1.24 \pm 0.14$ \\
2 & $9.91 \pm 0.48$ & $8.37 \pm 0.24$ & $3.18 \pm 0.23$ & $1.54 \pm 0.36$ \\
3 & $10.1 \pm 0.21$ & $8.63 \pm 0.06$ & $2.70 \pm 0.55$ & $1.47 \pm 0.14$ \\
4 & $10.2 \pm 0.25$ & $8.86 \pm 0.41$ & $2.98 \pm 0.31$ & $1.33 \pm 0.21$ \\
5 & $9.73 \pm 0.83$ & $8.74 \pm 0.85$ & $3.06 \pm 0.32$ & $0.99 \pm 0.84$ \\
6 & $10.26 \pm 0.29$ & $9.19 \pm 0.18$ & $2.32 \pm 0.16$ & $1.07 \pm 0.24$ \\
7 & $9.05 \pm 0.20$ & $8.35 \pm 0.11$ & $3.16 \pm 0.08$ & $0.7 \pm 0.16$ \\
\hline
\end{tabular}

The loss of horizontal velocity includes the loss of the conversion speed and the loss of the destructive running speed. The ratio between the two is determined by the athlete's quick support ability and rapid kicking ability. The stronger the quick support ability is, the faster the kicking ability is. The greater the proportion of the loss rate of the conversion type run-up speed is, and vice versa.

As shown in figure 1, Shandong athletes takeoff the average speed and excellent athletes in the world, compared to the average speed of evident when they accelerated last horizontal velocity of the phase jump pedal instantaneous horizontal velocity is small, jump off the ground instantly vertical velocity has a big gap with the world advanced level. In the eight subjects, four of the athletes had a greater rate of loss of velocity, while the vertical speed did not increase correspondingly. The cause of this situation is: 1. blindly pursuit of the horizontal speed. The research indicates that the average speed utilization rate of excellent athletes in China has reached $98 \%$, which is far higher than the $93 \%$ of the world's elite athletes [8]. This kind of high speed utilization rate is bound to affect the vertical velocity transformation. 2 . The lack of athletes' strength, especially the lack of power in the lower extremity, caused the athlete's body to not fully rise during the extension stage. 3. The transition time of the athletes is too short, so that the increase and transformation of the vertical velocity is not big enough.

The effect of buffering is not only based on the joint angle of the jump, but researching the reasonable range of joint angle has certain positive significance for the improvement of jumping technology. In the moment of jump, the athlete will take the initiative to knee buffering due to the reaction of the ground to the jump leg, which is the self-protection of athletes and also the main source of the vertical speed.

Buffering is the basis and premise of stretching, in order to improve the jumping effect, we must first improve the buffering effect. But at this time the knee angle can not be too large, too large, indicating insufficient strength athletes knee, poor cushioning ability, will make the buffer time increases, increasing the speed loss. Kick off the ground from the knee angle is small, indicating lack of muscle strength around the knee, kicking is not sufficient, affecting the vertical velocity increases; knee angle, turn the hip, knee and ankle in turn correct force of the three joints, knee circumference muscular strength, pedaling stretch full and powerful, to create more favorable conditions for the jump. These athletes knee angle was significantly smaller, become an important factor affecting the results. It is not ideal for the jumping angle of Shandong athletes, so that the initial speed is limited. With regard to the rising angle, we can see from the formula of the inclined throw motion in mechanics that under the certain throwing speed, the best throwing angle is about $45^{\circ}$. However, the actual situation is difficult to match. The reason is that the human body can not do it at any angle while keeping its initial speed unchanged. During the take-off process, the rapid change of body momentum accompanying the influence of speed inevitably accompanies the decrease of energy. The larger the rising angle, the more the energy decreases, and hence the speed of the rising becomes smaller. But in a certain range, the larger the starting angle, the greater the initial velocity. 


\section{CONCLUSIONS AND RECOMMENDATIONS}

\section{A. Shandong athletes should accentuation their jumping} angle training

Five of the athletes from Shandong province have a slightly lower angle of the knee joint, while all the athletes have smaller jumping angle. Scientific training should be carried out to improve the concession contract of muscles in lower extremity take-off work, improve the mechanical effect of muscle contraction and contraction during take-off, and obtain the best starting angle.

\section{B. Shandong athletes should increase their vertical speed}

Shandong athletes should improve their ability to take-off fastly and improve their taking-off technology. In the premise of developing absolute speed, more fully prepare to take off, increase the proportion of the vertical speed in the initial speed, and thus create better results. Shandong athletes have a low jump level, four of them have a large rate of loss, and four other athletes have a gap in the vertical speed compared to the excellent athletes in the word.

\section{Shandong athletes should strengthen core strength training}

The core strength training will be added to improve the muscle strength of the waist and lower limbs, on the basis of strengthening the training of regular special strength, shorten the gap with excellent long jump athletes in the world.

\section{REFERENCES}

[1] Shuimin Zhu, Wulong Zhang. Discussion on the effective ways of jumping technology for men's long jump in China [J]. Journal of Beijing institute of sport, 1997, 9(4):53-56.

[2] Qingjun Pang. The last two steps and the kinematics analysis of the leapforward technique of the excellent men in the long jump of China and the United States [D]. Wuhan: graduate department, wuhan institute of physical education, 2006.

[3] Liangping Zhang, Xiaoya Lu. The research status of Chinese leapjumping technology [J]. Journal of neijiang normal university, 2006, 21(6): 112-116.

[4] Xianli Lu, Ni Zhang. A review of biomechanics of leap-jumping technology [J]. Journal of ankang, 2006, 18(5):72-75.

[5] Wei Feng, Baoquan Gao, Rongli Niu. The characteristics of height change of the body weight in the last two steps of the excellent men's long jump in China [J]. Journal of Beijing sports university, 2004, 27(9):1277-1278.

[6] Tong yanhua. Relationship between the loss of horizontal speed and the long jump. [J]. China sports technology, 1999,35(11):35-36.

[7] Xie Wu, Wenyi Wei.The biomechanical review of leap-jumping technology [J]. Sports research. 2005, 26(4):57-60.

[8] Yongzhan Ma, Ailian Fang. A comparative study on the kinematics of Chinese excellent long jump athletes. [J], China sports technology, 2001,32(7):22-24 